\title{
BÂTIR DES TERRITOIRES CULTUELS POUR CONSTRUIRE DES IDENTITÉS.
}

\author{
Par Olivier BAUER \\ Article paru dans Studies in Religion / Sciences religieuses - 37/2 - 2008 - pages 235-247
}

Adresse de contact : olivier.bauer@umontreal.ca

Le théologien protestant Olivier Bauer réfléchit sur la manière dont les actions liturgiques bâtissent des espaces cultuels et sur les identités qu'elles construisent ainsi. Ses réflexions sont nourries par son expérience des cultes sur 3 continents, en Europe, en Océanie et en Amérique du Nord. Dans une première partie, il détaille ce que font les officiants et les célébrants - célébrer, regarder, bouger, écouter, s'exprimer, toucher, manger, sentir... - pour faire apparaître les représentations de l'espace cultuel qu'elles créent. Dans une seconde partie, il met en évidence les conséquences de l'aménagement d'un territoire cultuel sur l'identité religieuse de celles et ceux qui le fréquentent: sur leurs relations à Dieu, à l'Église et au culte lui-même.

As a protestant theologian, Olivier Bauer explains how liturgical performances build liturgical spaces and what kind of identities they construct doing so. He feeds his thoughts by his experience of worship on three continents: Europe, Oceania and North America. In his first part, he precisely relates what both liturgists and people are doing during worship - celebrating, looking, moving, hearing, speaking, touching, eating, smelling... - in order to let appear the pattern of the liturgical space these actions create. In his second part, he emphases how the construction of a liturgical space affects the religious identity of those who enter in it, especially in their relation with God, with the Church and with the worship itself.

On exagérerait à considérer une église comme un pays, même si l'on connaît un État guère plus grand que l'église qui le constitue même si l'on sait que, jadis, les églises ont pu bénéficier d'une certaine extraterritorialité : lieu d'asile de fait sinon de droit. Et si certains y viennent en visite, si d'autres y séjournent un peu plus longtemps, il lui manque quand-même, pour être un véritable pays, quelques résidents permanents. Mais une église est un territoire - ou plutôt des territoires comme on le verra plus loin - dont la géographie exerce un effet sur celles et ceux qui y pénètrent, en particulier lorsqu'ils participent à la célébration qui s’y déroule.

C'est sur cet effet que j'aimerais réfléchir ici, dans une démarche de théologie pratique qui part de l'observation des temples - les protestants francophones aiment à utiliser ce terme - et des cultes que l'on y célèbre, en Suisse romande, en Polynésie française, en France, ainsi qu'en Amérique du Nord1.

Mais un rappel préalable s'avère nécessaire. Pour la théologie protestante, aucun lieu n'est cultuel par essence et tous le deviennent pour peu qu'ils hébergent un culte : un temple protestant, naturellement, mais aussi une église catholique, une salle familiale, une classe d'école, un gymnase, une place publique ou une clairière dans la forêt. Ici, points de vue catholique et protestant ne s'accordent donc pas. Joseph Ratzinger l'avait bien perçu lorsqu'il écrivait: «Le lieu de culte protestant est vivant lorsque la communauté y est réunie ; en dehors des heures de rassemblement, il est en soi un espace vide. Il n'a d'existence que pour le rassemblement, lié pour ainsi dire à la fonction de la communauté et de son activité liturgique. Par contre, là où l'on croit à la présence réelle durable, il y a toujours "eucharistie", l'espace n'est jamais vide» (Ratzinger 2003, 255). Le propos est exact. Catholique, la théologie installe Dieu dans des espaces, celui de l'église en premier lieu et par excellence. Protestante, elle lie Dieu aux personnes. «L'essentiel est l'assemblée ou l'Église et non pas un lieu ou l'église : la communauté elle

1 Pour d'autres réflexions sur le culte, je me permets de renvoyer à mes propres travaux : BAUER, O (2003). Les rites protestants polynésiens Quand faire c'est dire!, Paris : L'Harmattan ; BAUER, O (2008). Le protestantisme et ses cultes désertés Genève : Labor et Fides. 
seule, et non pas un bâtiment, définit la réalité ecclésiale et cultuelle en profondeur » (Gagnebin 1992, 31). En protestantisme, l'existence d'un espace cultuel dépend toujours de son emploi pour le culte. On parle ainsi de «lieu du culte», expression qui qualifie simplement l'endroit où un culte est célébré. Bien évidemment - mais peut-être est-ce utile de le rappeler? - que l'espace soit cultuel ou non ne détermine en rien la présence de Dieu en son sein. Dieu peut habiter tous les territoires, aussi bien le temple durant le culte que n'importe quel endroit à n'importe quel moment.

\section{Construire des territoires}

Un temple - comme tout lieu où un culte est célébré - connaît toujours au moins quatre bâtisseurs : l'architecte, les artisans et les artistes qui en dessinent, en construisent et en décorent les murs, le sol et le toit; la communauté qui en aménage l'intérieur; les activités qui l'occupent, enfin, le théologien qui transforme des bâtiments spécifiques en un modèle idéal, adéquat pour son propos.

À elle seule, l'analyse de l'architecture et de l'aménagement intérieur ne peut donc suffire à comprendre les territoires qui composent un temple. Car les aspects solides et matériels du bâtiment ne parviennent pas à fixer les territoires intérieurs, même s'ils y contribuent. Pour comprendre le rôle de l'aménagement du territoire cultuel dans la structuration des identités religieuses, il faut s'intéresser aux espaces construits par les cultes ainsi qu'aux fonctions que ceux-ci leur attribuent.

Mais, puisque tous les cultes et tous les lieux qui les hébergent sont différents, on devrait traiter chaque temple comme un cas unique, ce qui pose des problèmes évidents. Pour la commodité du propos, je vais donc reconstruire ici - par généralisation, par omission et sans éviter certaines déformations - un temple et un culte idéaux. Ni l'un ni l'autre n'existent nulle part au monde. Mais s'ils existaient, ils seraient typiquement protestants.

\subsection{Célébrer un culte}

Célébrer un culte, c'est accomplir des actions de deux types : expressives - parler, chanter, se déplacer, bouger, faire des gestes - et perceptives - voir, entendre, sentir, toucher et goûter2. Ainsi donc, même si les officiants prennent en charge la plupart des actions d'expression, ils ne sont jamais les seuls acteurs du culte. Les participants s'expriment peu, mais perçoivent beaucoup. C'est toujours ensemble que les célébrants - j'appelle ainsi la réunion des officiants et des participants - construisent le culte par des actions que l'on dira « liturgiques», au sens étymologique du terme : un « faire du peuple» au « service du peuple». Car il faut s'y résoudre. On aimerait que Dieu agisse dans le culte ou dans la messe, mais ce ne sont que des êtres humains qui le ou la célèbrent. Comme l'indique Manfred Josuttis : «Toute forme de pratique cultuelle renferme une structure d'action paradoxale. On doit faire quelque chose dans un domaine de la vie dans lequel, par définition, aucun être humain ne peut rien faire » (Josuttis 1991, 104). Nous ne pouvons rien, mais nous devons tout.

On ne peut sortir de cette impasse qu'en reconnaissant que les actions liturgiques sont toutes, toujours, des actions humaines. Et c'est pourtant, par elles, que les célébrants cherchent à exprimer une relation avec Dieu - partant, avec soi-même, avec les autres, avec le monde - celle qu'ils estiment évangéliquement juste. Ils choisissent des actions liturgiques - des actions d'expression et de perception - pour faire percevoir Dieu, par les oreilles, les yeux, la langue, le nez, la peau et les muscles.

2 Sur la manière dont le culte protestant sollicite ou non les cinq sens, je me permets encore une fois de renvoyer à mes recherches : BAUER, O (2001) «L'essentiel est inaudible aux oreilles », Études Théologiques et Religieuses 76/2 : 213-227 (disponible librement à l'adresse http://hdl.handle.net/1866/785); BAUER O (2004). «Du beau, du bon... du bon Dieu », Lumen Vitae, 2 : 149-159 (disponible librement à l'adresse http://hdl.handle.net/1866/778). 
Chacune des actions et chacune des perceptions pourraient faire l'objet d'une recherche particulière : quel est le «goût» d'un Dieu que l'on rencontre dans l'hostie ou dans le pain levé ? quelle est la "stature » d'un Dieu que l'on prie debout, assis ou à genoux ?

Dans cet article, je vais m'attacher à préciser comment les actions liturgiques construisent des territoires à l'intérieur du lieu du culte, comment elles leur attribuent des fonctions et comment elles structurent ainsi les identités religieuses de celles et ceux qui les accomplissent. Nous verrons que les perceptions du regard, du mouvement, de l'ouie, de la parole, du tact, du goût et de l'olfaction sont souvent redondantes, mais qu'elles apportent parfois des informations différentes, voire contradictoires.

\subsection{Regarder}

Les yeux divisent le paysage en deux territoires distincts : le lieu du culte d'un côté et « le reste du monde » de l'autre, le temple d'une part et « tout ce qui n'est pas le temple » de l'autre. Car l'existence d'un espace cultuel signifie forcément celle d'un autre espace qui n'est pas cultuel. À l'extérieur, la différence entre l'un et l'autre est rendue visible par les murs du temple, par le parvis, le jardin, le mur ou la barrière qui l'entourent; à l'intérieur, elle se remarque notamment à la pénombre ou à la lumière colorée des vitraux.

Les yeux de celui qui pénètre dans le temple construisent une salle unique, partagée en plusieurs territoires, délimités par des frontières plus ou moins visibles : quelques marches, une barrière, des revêtements de sol différents, un plancher ou un carrelage dont les motifs varient. Si l'on remarque parfois plusieurs territoires distincts - espaces d'accueil, de prière, d'information ou d'exposition -, on en voit toujours au moins deux que l'aménagement distingue : la partie la plus grande est remplie de bancs, c'est le territoire des participants ; la plus petite est presque vide, à l'exception de quelques meubles, c'est le territoire des officiants. La géographie des territoires intérieurs propose deux grands modèles, ceux que Bernard Reymond appelle « dispositif en long » et " quadrangle choral réformé » (Reymond, 1996, 142-147). Dans le premier, le bâtiment est utilisé dans sa longueur et les deux territoires - chœur et nef selon la terminologie classique - se font face. Dans le second - plus typiquement protestant -, le bâtiment est utilisé dans sa largeur : les bancs sont disposés en forme de « $U$ » sur trois côtés, le centre du quatrième toujours une longueur - étant réservé aux officiants. L'amphithéâtre - fréquent dans les megachurches aux États-Unis - représente encore une autre possibilité, voie médiane entre les deux premiers aménagements : si les bancs sont disposés en arc de cercle, ils font cependant tous face à une scène où officient les musiciens et le prédicateur. Dans les trois dispositifs, le centre du territoire des officiants est occupé par la chaire et la table de communion, flanquées des fonts baptismaux et d'un pupitre ou d'un lutrin.

Les yeux remarquent encore un orgue, monument presque inévitable dans le paysage des églises chrétiennes. Installé parfois dans le territoire des officiants, il est fréquemment placé chez les participants, de préférence dans leur dos.

\subsection{Se déplacer, adopter une posture, faire des gestes}

Les pieds font du temple un territoire spécifique. Car participer au culte implique un déplacement. Il faut se lever, quitter sa maison et se rendre jusqu'au lieu du culte. Les célébrants doivent encore traverser le parvis, la porte et le vestibule - le narthex dans la terminologie traditionnelle- pour pénétrer dans l'espace cultuel. Les participants s'arrêtent dans la nef, mais les officiants poursuivent leur chemin jusque dans le chœur ou ce qui en tient lieu.

La célébration de la cène augmente les déplacements. Certaines communautés la célèbrent dans le chœur : à l'invitation des officiants, les participants doivent quitter leur territoire et former un cercle dans le territoire des officiants pour recevoir le pain et le vin. D'autres paroisses l'organisent dans la nef : ce sont alors les officiants qui doivent quitter leur territoire et se rendre " chez les participants » pour donner le pain et vin aux communiants qui défilent devant eux. Les officiants restent parfois à la limite des deux territoires, construisant ainsi une sorte de zone franche entre les deux. Mais ils pénètrent aussi vraiment 
dans le territoire des participants, soit que les officiants proposent plusieurs points de communion, soit que les célébrants reçoivent le pain et le vin «dans les bancs ", là où ils sont assis, comme cela se fait en Polynésie française, lorsque l'assemblée est nombreuse.

Autre mouvement, en Polynésie toujours, au Cameroun aussi, ailleurs sûrement, les officiants demandent parfois aux participants de venir déposer l'argent de leur offrande sur une table, dans cette zone franche qui sépare le territoire des participants et celui des célébrants.

À la fin du culte, officiants et participants refont à l'envers le chemin qui les a conduits au culte. Ils sortent du temple, s'arrêtent parfois quelques instants sur le seuil et retournent « dans le monde » pour rentrer chez eux.

Tout au long du culte, les participants restent assis. Ils ne se lèvent que pour chanter, pour dire la confession de foi et pour écouter quelques textes liturgiques: invocation, annonce du pardon et bénédiction. Les officiants se tiennent debout tant qu'ils parlent.

Les gestes, utilitaires ou symboliques, sont rares dans le culte protestant : les participants joignent les mains pour prier ; ils prennent, tiennent et donnent l'argent pour l'offrande ; les officiants rompent le pain et élèvent la coupe au moment de la cène ; ils étendent les bras au moment de la bénédiction.

\section{4. Écouter, parler, chanter, rester silencieux}

Tout ce qui est visible occupe une place bien déterminée. Mais les bruits et les sons se diffusent. Ils ne sont liés à aucun lieu, même s'ils ont une source identifiable. Les cloches par exemple sonnent dans le clocher, mais remplissent le temple et tout son voisinage. Au cours du culte, les oreilles construisent des territoires compliqués aux frontières changeantes, qu'il vaut la peine de présenter avec soin.

Lorsque les oreilles entendent les mots prononcés, le ton sur lesquels ils sont dits, les musiques jouées, elles distinguent le temple du monde qui l'entoure. Car ni les mots du culte, ni ses mélodies, ni le rythme des paroles et des musiques ne sont ceux de la culture contemporaine.

Lorsqu'elles écoutent les locuteurs, les oreilles divisent le temple en deux territoires. Les officiants habitent un territoire où il est permis de beaucoup parler, de parler au nom de Dieu - annonce du pardon et bénédiction, lectures bibliques et prédication -, de parler à Dieu - dans les moments de prières - et de parler des célébrants aux célébrants - au moment de transmettre des informations sur la vie de la communauté.

Les oreilles les plus fines distinguent encore, dans le territoire des officiants, trois lieux auxquels correspondent trois types d'expression : depuis un lutrin, les officiants lisent les mots traditionnels de la liturgie ; depuis la chaire, le prédicateur prononce des phrases originales qu'il a imaginées ; et derrière la table de communion, les officiants doublent les mots de l'institution de la cène par les gestes symboliques de la fraction du pain et de l'élévation de la coupe de vin. De leur côté, les participants héritent d'un territoire où ils parlent peu, où ils parlent essentiellement à Dieu - dans les prières -, parfois à la communauté quand il s'agit de transmettre des informations.

La récitation du «Notre Père » ou d'une confession de foi, les rares moments de silence réunissent les uns et les autres dans un espace unique. Et la musique supprime elle aussi les frontières. Elle le fait de trois manières différentes : elle réunit tous les célébrants pour chanter en chœur; elle permet aux participants de transmettre la Parole de Dieu, lorsqu'ils chantent des psaumes ou des cantiques bibliques; elle fusionne enfin le territoire des officiants et celui des participants. Car les musiciens, qu'ils soient placés dans l'un ou l'autre territoire, sont tantôt des participants, lorsqu'ils accompagnent le chant de la communauté, tantôt des officiants, quand ils jouent seuls. Ils sont tantôt des officiants installés chez les participants, tantôt des participants qui habitent chez les officiants. 


\subsection{Toucher, être touché}

La peau, par les sensations tactiles, contribue à faire du temple un «territoire à part », lorsqu'elle perçoit les contrastes de températures entre l'extérieur et l'intérieur du lieu de culte. C’est elle aussi qui construit le territoire intermédiaire entre le temple et le monde.

L'endroit où les célébrants se serrent la main ou s'embrassent - essentiellement le parvis et l'entrée - forme une sorte de sas entre l'extérieur et l'intérieur, un espace qui n'est plus vraiment le monde, mais pas encore le temple, plus vraiment le temple, mais pas encore le monde.

Enfin, la peau des baptisés, des confirmés et des personnes dont le mariage est béni leur fait sentir qu'ils sont devenus citoyens du pays des officiants puisque c'est dans ce territoire que l'eau du baptême ou les mains des officiants touchent leur tête.

\subsection{Manger, boire}

Au cours du culte proprement dit, le goût n'est sollicité que lorsque la cène est célébrée. La fréquence en est variable : d'une fois par mois à chaque dimanche. Suivant la manière de procéder, la langue peut alors construire trois territoires différents. Que tous les célébrants se réunissent autour de la table de communion pour manger le pain et boire le vin et la langue abolit la frontière qui délimite le territoire des officiants. Que les officiants apportent le pain et le vin chez les participants et la langue transforme le territoire des participants en un lieu où l'on officie. Mais que la communion se déroule dans la «zone franche » et la langue maintient la séparation entre les deux territoires.

Les communautés offrent souvent un apéritif après le culte. Lorsqu'il est servi sur le parvis, la langue souligne alors le rôle du sas entre le temple et le monde. Et lorsqu'il est servi à l'intérieur, la langue perçoit que le temple sert aussi à renforcer les relations entre les célébrants.

\subsection{Sentir}

Les frontières n'arrêtent pas plus les odeurs que les sons. Celles-la aussi, à partir de l'endroit d'où elles sont émises, remplissent les volumes. Il est donc difficile de leur attribuer un territoire précis.

Les murs et les fenêtres fonctionnent comme un puissant filtre qui isole le lieu du culte À l'intérieur du temple, le nez construit un territoire unique, supprimant les frontières. De toute façon, s'il n'en tenait qu'au nez, le territoire cultuel existerait à peine. Car le culte protestant ne sollicite pas activement l'odorat. Le nez peut néanmoins saisir quelques odeurs : les parfums des célébrants, celui du bois dont sont faits les bancs ou les murs, ceux des produits d'entretien, éventuellement ceux des fleurs et des plantes, le cas échéant les odeurs du pain et du vin de la cène.

\subsection{Le territoire cultuel}

Il faut prendre acte de ce double constat : en théologie protestante, aucun lieu n'est cultuel en soi ; chaque culte construit l'espace en fonction du bâtiment, de son aménagement intérieur et de la manière dont la célébration se déroule.

Les actions liturgiques protestantes font du lieu du culte un territoire indépendant du monde qui l'entoure, tantôt réunis en un seul espace, tantôt divisé en deux régions distinctes : le territoire des participants et celui des officiants. La célébration du culte définit deux zones intermédiaires : un «sas » entre le monde et le temple, une « zone franche » entre les territoires des participants et celui des officiants.

\section{Structurer les identités religieuses}

Avec les matériaux ressemblés dans l'observation, il est maintenant possible d'expliquer comment l'aménagement du territoire cultuel structure l'identité religieuse de celles et ceux qui le fréquentent. Je vais m'intéresser plus spécialement à trois types de rapports qu'induisent les actions liturgiques qu'elles soient expressives ou perceptives : rapports à Dieu, à l'Église et au culte lui-même. 


\subsection{Rapport à Dieu}

Commençons par signaler qu'un temple rappelle l'existence d'une Église, et probablement celle de Dieu, à toutes celles et ceux qui le voient, y compris les membres de religions non-chrétiennes et les noncroyants.

Conçu par des théologiens protestants, le temple n'est jamais un territoire sacré. Mais bâti par des artisans et des artistes, il est forcément un bâtiment unique. Construit par les actions liturgiques, le territoire cultuel reflète cette ambivalence : certaines perceptions en accentuent le caractère spécifique - c'est le cas notamment de la vue, de l'ouie et des déplacements -, d'autres tendent à le confondre avec le monde qui l'entoure - essentiellement l'olfaction et le goût. Nous allons les examiner tour à tour.

Qui veut participer au culte se met en route pour se rendre au temple, comme il peut partir en voyage. Il passe la frontière que forment le parvis et l'entrée. Il se retrouve alors dans un territoire étranger - parfois très étrange -, qui ne ressemble pas à celui qu'il vient de quitter. L'architecture est particulière, les symboles aussi. La décoration, la luminosité, les sièges où l'on s'assied, les vêtements des officiants, la langue que l'on y parle, la musique que l'on y entend, même le ton qu'adoptent les orateurs, tout concourt à donner l'impression que le culte se déroule dans un territoire qui lui est propre, un territoire autonome, parfois franchement exotique.

Et pourtant d'autres actions liturgiques produisent l'effet inverse : aller au culte, ce n'est plus partir au loin, mais se retrouver un peu chez soi. Ainsi, l'odeur du temple est celle de tous les bâtiments qui lui sont contemporains. Le pain et le vin de la communion sont des aliments ordinaires, semblables à ceux que chacun peut acheter dans sa boulangerie ou dans son épicerie. Les gens que l'on rencontre et que l'on salue sont des voisines et des voisins. Le lieu du culte peut être aussi familier qu'un salon ou une salle de classe.

Ce double aspect du temple protestant - territoire à la fois exotique et familier - me conduit à discuter son éventuel caractère sacré. En protestantisme, tout le sacré est concentré en Dieu, et rien de ce qui appartient à la création, ni la nature, ni l'être humain, ni aucune de ses réalisations - même les meilleures, même les plus belles - ne le sont. Le lieu du culte, temple compris, n'échappe pas à ce principe. Il n'est pas un territoire sacré. Ou alors il l'est au sens d'un territoire "mis à part », utilisé pour favoriser et faciliter le contact avec Dieu. Et cette «sacralisation» fonctionne comme le tapu des Polynésiens : «Le tapu [...] n'est jamais une valeur absolue; le tapu est moins tapu en soi que tapu par rapport à quelque chose d'autre. Une personne par exemple tapu, mais sa tête est l'endroit le plus tapu de son corps » (Arnoux 1993, 42). Le temple n'est pas un territoire sacré en lui-même. Mais, traditionnellement, le lieu du culte est plus « sacré » que le monde qui l'entoure, le territoire des officiants est plus « sacré » que celui des participants et la chaire et la table de communion plus «sacrées » que le chœur. Mais le taux de sacralisation et sa distribution à l'intérieur du bâtiment ne sont pas fixes. Ils varient selon la manière dont l'espace dévolu au culte est bâti, aménagé et utilisé.

Le « sas » entre le temple et le monde joue ici un rôle important. Il peut rendre étanche le lieu du culte, en empêchant l'extérieur d'y pénétrer, mais il peut aussi représenter un point de passage qui facilite les échanges entre le monde et le temple, entre le temple et le monde. Et la «zone franche » remplit la même double fonction entre le territoire des participants et celui des officiants. Choisir de renforcer la frontière entre le temple et le monde, interdire aux participants de pénétrer dans le territoire des officiants, c'est renforcer le caractère «sacré » des territoires du culte et des officiants. À l'inverse, célébrer le culte dans une salle de classe et renforcer les interactions entre tous les célébrants, c'est désacraliser le lieu du culte et les espaces intérieurs.

On peut même imaginer un bâtiment construit pour célébrer le culte qui soit complètement désacralisé. De prime abord, une telle réalisation semble paradoxale puisqu'il s'agit de délimiter un territoire spécifique tout en refusant de le distinguer du monde qui l'entoure, puisqu'il en va d'aménager un lieu propice à la célébration sans réserver un territoire aux officiants. Mais la logique théologique protestante est ainsi aboutie : tout le sacré étant en Dieu, il n'est pas plus dans la chaire ou derrière la table de communion que 
partout ailleurs dans le temple, pas plus dans le temple que n'importe où dans le monde. Reste à le démontrer en construisant et en aménageant un espace cultuel désacralisé !

Autrement dit, les protestants aiment que le culte se déroule dans un lieu identifiable, symbolique et pratique, ce qui explique la prédilection à le célébrer dans un temple ou une église, lorsque c'est possible. Mais en même temps, le lieu du culte ne doit pas paraitre trop religieux, d'où l'utilisation de salles de classe ou de salle familiale. La désacralisation du territoire cultuel s'inscrit dans la volonté de témoigner de la présence de Dieu dans les choses ordinaires : le pain et le vin de la communion sont des aliments communs dont les éventuels restes sont souvent consommés en guise d'apéritif après le culte ; l'eau du baptême vient du robinet et sera jetée après usage; le pasteur est un homme ou une femme ordinaire auquel la communauté attribue simplement une fonction particulière, etc.

\subsection{Rapport à l'Église}

Les actions liturgiques permettent de structurer le rapport que chacun peut entretenir avec une Église. Elles précisent en quelque sorte ce que signifie être citoyen du territoire cultuel. L'identité religieuse qu'elles transmettent incorpore tout à la fois la structure hiérarchique de l'Église et le principe du sacerdoce universel.

La plupart des perceptions fait distinguer deux territoires à l'intérieur du lieu du culte et les différents sens construisent des démarcations, qui se recouvrent souvent : l'espace où l'on s'assied est aussi celui où l'on écoute ; l'espace d'où l'on parle est celui où l'on se tient debout. Mais les frontières ne se superposent pas toujours : l'espace des officiants, par exemple, n'est pas le seul endroit d'où est transmise la Parole de Dieu. Il vaut donc la peine d'apporter quelques précisions.

La vue divise l'espace en deux territoires plus ou moins imbriqués, séparés par une frontière plus ou moins marquée. En règle générale, le "dispositif en long» tend à accentuer la séparation, tandis que le « quadrangle choral » l'atténue. De manière générale, les officiants vivent dans un territoire presque vide, à l'exception de quelques sièges, d'un pupitre ou d'un lutrin et d'une table derrière lesquels ils se tiennent debout. Les participants habitent eux dans une région remplie de bancs sur lesquels ils doivent s'asseoir.

La bouche et les oreilles confirment cette partition du lieu du culte. La Parole de Dieu habite surtout le territoire des officiants - qui peut se réduire au seul pupitre - d'où se font la lecture de la Bible, la prédication, l'annonce du pardon, la bénédiction, toutes paroles dites au nom de Dieu. Mais on y dit aussi des paroles à Dieu et l'on y fait les annonces à la communauté. Dans le territoire des participants, on s'adresse à Dieu dans les prières, on se dit devant Dieu dans la confession de foi, on transmet la Parole de Dieu dans les chants.

Les pieds de celui ou de celle qui prend part au culte précisent le statut que la communauté lui reconnaît. Car si tous les célébrants franchissent la frontière et pénètrent dans le territoire du culte, seuls les officiants s'installent dans le chœur ou dans l'espace qui en tient lieu. Et l'on peut encore affiner la distinction, puisque certains officiants s'arrêtent au lutrin, pour lire la liturgie, tandis que d'autres montent en chaire pour proclamer leur prédication ou vont derrière la table pour célébrer la cène. Ici s'applique le principe du «qui peut le plus, peut le moins »: le pasteur peut toujours s'asseoir sur un banc chez les participants ou parler au lutrin. Mais l'inverse n'est pas vrai. Sans mandat spécifique, un participant ne peut pas s'inviter dans le territoire des officiants.

Diviser le lieu du culte en territoires, y situer certains types de discours et limiter l'accès à certains lieux, c'est se donner les moyens de préciser le statut des célébrants - participants ou officiants - et d'indiquer la hiérarchie parmi les officiants : dans l'ordre croissant, probablement, lecteur, liturge - avec une valeur particulière pour celles et ceux qui sont habilités à célébrer la cène -, prédicateur.

J'ai souligné comment le regard, les déplacements et l'écoute partagent le lieu du culte en deux territoires. Il ne faudrait pas pour autant négliger ce fait évident : le temple protestant est, dans son entier, la patrie de tous les célébrants, officiants et participants. 
L'ouie, l'olfaction et le goût abolissent les frontières et reconstruisent cet espace unique. Ces trois sens affirment ainsi le principe du sacerdoce universel. Le chant réunit participants et officiants pour transmettre la Parole de Dieu. En remplissant tout le temple, les odeurs font du lieu du culte un territoire unique. Et le goût réunit tous les célébrants pour boire et manger la Parole de Dieu dans un espace unique, quand les participants accèdent au territoire des officiants ou quand les officiants se rendent dans celui des participants. Mais célébrer la communion dans la «zone franche », c'est reconstruire - ou maintenir - la séparation entre les officiants et les participants.

Le toucher enfin supprime la frontière, mais pour un temps seulement et uniquement pour quelques personnes. Car c'est en général dans le chœur que les officiants versent de l'eau sur la tête des baptisés, qu'ils posent leurs mains sur celle des confirmands et des mariés, rares moments de " toucher liturgique » dans le culte protestant.

Il est évident que l'invitation faite aux participants de pénétrer dans le territoire des officiants pourrait être interprétée comme une « désacralisation » du chœur. Il me semble pourtant, à voir avec quel respect les participants y viennent et s'y tiennent, que ce déplacement produit l'effet inverse : il «sacralise» les participants en les faisant accéder au rang des officiants.

\subsection{Rapport au culte}

Pour terminer ce bref aperçu, j'aimerais m'asseoir un moment sur l'un de ces bancs qui remplissent les temples. Je suis conscient de m'éloigner un peu de la notion de territoire au sens strict. Mais je veux m'intéresser aux conditions de vie que les participants rencontrent dans leur territoire.

Difficiles à déplacer, les bancs ne sont certes pas pratiques. Mais en dépit ou en raison de leur inconfort manifeste, dans leur banalité, ils sont caractéristiques des temples et remplissent des fonctions cultuelles.

Les bancs d'abord sont vus. Au regard, ils indiquent le rôle fondamental que les participants jouent dans le culte, ils construisent la dimension horizontale du temple. C'est particulièrement évident dans le dispositif appelé «quadrangle choral». Disposés sur trois côtés, mettant les participants face à face, les bancs démontrent que le culte ne cultive pas seulement le rapport à Dieu mais qu'il concerne aussi les relations entre les êtres humains. Accessoirement, parce que beaucoup restent vides, ils rappellent aussi - cruelle réalité pour celles et ceux qui participent au culte - la déchristianisation de nos sociétés.

Mais ces bancs « disent» encore plus. Car on ne se contente pas de les regarder, on s'y assied. Et le simple fait d'être assis détermine le rapport que l'on entretient avec le culte. Il faut s'en souvenir, les chrétiens ne se sont pas toujours assis, et aujourd'hui encore, ils ne s'assoient pas tous pour participer au culte. De la même manière que les orthodoxes aujourd'hui, les chrétiens occidentaux ont longtemps célébré la messe debout, pouvant quitter l'église quand ils le souhaitaient. S'asseoir demeurait le privilège de l'évêque. C'est au Moyen Âge que les bancs furent introduits dans les églises donnant aux participants la possibilité ou plutôt l'obligation de s'asseoir. L'adoption de la position assise ainsi que le fait de devoir assister à toute la messe a donné aux célébrations du christianisme occidental une dimension clairement intellectuelle ou pédagogique, à la fois pour des raisons symboliques - l'église ressemblant à l'école - et pour des raisons physiologiques - la position assise facilitant la concentration et la réflexion. En faisant de la prédication, le centre du culte, la Réforme n'a fait qu'accentuer cette tendance. On peut affirmer avec Ralph Kunz que «le processus de protestantisation a rendu le culte plus verbal et plus littéraire (Kunz 2001, 207). Assis sur leurs bancs, les participants au culte ressemblent à des élèves qui écoutent - ou entendent, selon les cas - l'enseignement de leur maitre.

Au toucher enfin, les bancs offrent peu de confort. Ils sont plutôt durs, peu ergonomiques; ils n'ont le plus souvent pas d'accoudoirs, ni rien qui permette d'être séparé de son voisin. Seul avantage à mettre à leur crédit, ils sont fabriqués en bois et restent donc d'une température constante : ils ne sont jamais froids en hiver et ne gardent pas la chaleur de la personne qui vient de quitter sa place.

Les bancs contribuent donc à structurer l'identité religieuse de celles et ceux qui participent au culte. Mettre des sièges dans un temple, c'est annoncer qu'il rassemble une communauté. Y faire asseoir les 
participants, c'est solliciter leur intelligence pour comprendre ce qu'ils font et ce qu'ils perçoivent. Choisir des bancs en bois, c'est négliger le confort pour obliger les participants à une certaine ascèse, très relative, je l'accorde. Le choix des sièges possède donc une forte dimension symbolique. Ainsi, lorsque des temples - c'est souvent le cas des megachurches américaines - proposent des fauteuils moelleux, elles augmentent certainement le plaisir que l'on peut prendre à participer au culte. Mais à voir comme à s'asseoir, elle les fait plutôt ressembler à des salles de cinéma. On court alors le risque de transformer les participants en auditeurs-spectateurs.

\section{Conclusion}

Ce court voyage dans les territoires cultuels protestants a permis d'en découvrir les aspects les plus significatifs. Je décris brièvement quelques-unes des curiosités que notre excursion nous a fait découvrir.

Le temple protestant forme un territoire spécifique, distinct du monde extérieur sans lui être étanche. Il est subdivisé en plusieurs territoires. Si les frontières sont fixées par l'architecture et par l'aménagement intérieur, elles sont aussi construites par les actions liturgiques qui font le culte. Elles sont donc mobiles puisqu'elles dépendent de ce que perçoivent chacun des cinq sens.

La célébration du culte divise le temple en plusieurs territoires et leur attribue des fonctions particulières. Elle tend à construire deux territoires, l'un pour les officiants et l'autre pour les participants. Mais la division n'est pas figée. Si la vue accentue la séparation, le goût tend à reformer un territoire unique « appartenant » à tous les célébrants.

L'aménagement des territoires cultuels structure les identités religieuses des gens qui les fréquentent. Elles colorent la relation qu'ils entretiennent avec Dieu - qu'ils peuvent rencontrer dans les choses ordinaires , avec l'Église - qui ne réserve pas l'annonce de la Parole de Dieu aux seuls officiants - et avec le culte lui-même - qui mobilise l'intelligence.

En guise de conclusion, je rappelle que les théologiens sont les quatrièmes constructeurs du temple. Personne ne s'étonnera donc que ni le culte ni le temple que j’ai présentés ici n’existent nulle part. Mais si j'assume qu'ils soient mon œuvre, je revendique aussi de les avoir construit avec les matériaux que j'ai pu récolter dans de nombreux temples, au cours de nombreux cultes.

\section{Bibliographie}

ARNOUX, Rosemary. (1993). "La tradition maaori, un monde perdu aux sources intarissables », in Magie et fantastique dans le Pacifique, Sylvie ANDRÉ (éd.), Papeete : Haere Po no Tahiti.

BAUER, Olivier. (2001). «L'essentiel est inaudible aux oreilles », Études Théologiques et Religieuses 76/2 : 213227 (disponible librement à l'adresse http://hdl.handle.net/1866/785)

BAUER, Olivier. (2003). Les rites protestants polynésiens Quand faire c'est dire !, Paris : L'Harmattan

BAUER Olivier. (2004). «Du beau, du bon... du bon Dieu », Lumen Vitae, 2 : 149-159 (disponible librement à l'adresse http://hdl.handle.net/1866/778).

BAUER, Olivier. (2008). Le protestantisme et ses cultes désertés, Genève : Labor et Fides.

GAGNEBIN, LAURENT. (1992). Le culte à chour ouvert, Genève : Labor et Fides.

JOSUTTIS, Manfred. (1991). Der Weg in das Leben, Eine Einfübrung in den Gottesdienst auf verhaltenswissenschaftlicher Grundlage, München : Chr. Kaiser.

KUnZ, Ralph. (2001). Gottesdienst evangelisch reformiert, Zürich : Pano.

RATZINGER, Joseph. (2003). "Réponse à la lettre ouverte d'Olivier Bauer », Revue de théologie et de philosophie, $135: 253-256$.

REYMOND, Bernard. (1996). L'architecture religieuse des protestants, Genève : Labor et Fides. 